\title{
Diversité culturelle et éducation au vivre ensemble en EPS : quelles compétences interculturelles pour un enseignement contextualisant?
}

\author{
Cheikh Tidiane-Wane et Laëtitia Cordier
}

Cheikh Tidiane-Wane.

Laëtitia Cordier.

Résumé. La France, comme bon nombre de pays européens, est confrontée à une diversité culturelle, religieuse et linguistique croissante dans les établissements scolaires. Il en résulte une coexistence, non sans difficulté, de diverses conceptions du monde, de la vie, de l'éducation. Pour autant les institutions scolaires doivent gérer cette diversité mais également favoriser la construction d'une culture commune en préparant de futurs citoyens capables de vivre ensemble et de prendre part à la vie de la cité. L'objectif de ce travail est donc d'identifier, à travers une étude de cas, les représentations du vivre ensemble qu'ont des enseignant-e-s d'EPS et les compétences interculturelles qu'ils mobilisent pour le favoriser en EPS en contexte interculturel. Les résultats montrent que le rapport au vivre ensemble des enseignants est lié à leur perception du contexte et aux valeurs qui font sens pour eux. Ces dernières déterminent et structurent les compétences interculturelles qu'ils mobilisent.

Mots-clés. Vivre-ensemble, Diversité, Interculturel, Compétences, Représentation

\section{Introduction}

Avec les réformes des programmes du collège (2015) et du lycée (2019) en France, la notion de vivre-ensemble a pris une place importante dans les finalités éducatives. « Tout au long de la scolarité, l'Éducation Physique et Sportive (EPS) a pour finalité de former un citoyen lucide, autonome, physiquement et socialement éduqué, dans le souci du vivre ensemble » (Ministère de l'Éducation Nationale, de l'Enseignement supérieur et de la Recherche, 2015). Ce souci du vivre ensemble, présent dans les discours sur l'éducation et la citoyenneté, depuis un certain nombre d'années s'est renforcé pour faire face aux défis du pluralisme culturel, religieux dans la société française. Il vise également à répondre aux tensions liées aux attentats (Charlie Hebdo, Bataclan, ainsi que l'assassinat d'un professeur d'histoire et de géographie récemment), qui jettent le trouble sur les capacités de cohésion nationale du pays et l'adhésion de tous aux valeurs républicaines, enseignées historiquement à l'école (Lorcerie, 2016). L'expression « vivre-ensemble » est polysémique et, selon Duhamel (2010), souvent utilisée pour caractériser un projet de société où l'espace commun se doit d'être harmonisé, en particulier par le dialogue. La notion de vivre ensemble n'est jamais définie de manière claire et précise. Elle dépend du contexte social et institutionnel. Pour mieux comprendre les enjeux autour du vivre ensemble, nous nous appuyons sur les travaux de Duhamel et Estivalèzes (2013). Ces auteurs, afin de mieux saisir ces multiples significations, ont proposé cinq sens de l'expression «vivre ensemble», selon les différents contextes dans lesquels elle est utilisée : l'expression peut désigner (1) une réalité sociale ; (2) un cadre normatif qui vise à favoriser la cohésion sociale dans une société plurielle; (3) un cadre éthique qui se traduit dans le paradigme de la reconnaissance de l'autre; (4) un projet politique de quête de valeurs communes, ou enfin; (5) une pratique dialogique aux vertus éducatives. Ces différentes acceptations montrent que cette notion possède un sens plastique et malléable qui se structure avant tout dans le contexte de ses occurrences et en fonction des autres concepts auxquels elle s'associe.

Dans le contexte de la France, Dubet (2014) précise que le vivre-ensemble c'est construire librement, dans un processus dynamique et continuel, son identité en adhérant à un projet collectif. En d'autres termes, le vivre-ensemble met en jeu des valeurs de liberté, égalité et fraternité. Il apparaît comme rempart contre le communautarisme, l'intolérance ou bien l'irrespect. A ce titre, Lamamy-Echard (2016) souligne que le vivre ensemble est à la fois un moyen et un objectif devant l'hétérogénéité du public scolarisé, dans un contexte où les phénomènes de violences, de tensions sociales, 
Diversité culturelle et éducation au vivre ensemble en EPS : quelles compétences interc...

d'agressions physiques et psychologiques sont récurrents. L'auteure souligne quatre piliers sur lesquels se fondent le vivre-ensemble : l'individu, la classe, le groupe et l'école. L'individu construit son identité avec et pour le groupe. En EPS pour atteindre la finalité de former un citoyen lucide, autonome, physiquement et socialement éduqué, dans le souci du vivre ensemble, le programme de formation de l'école française est organisé autour de cinq domaines du socle commun de connaissances, de compétences et de culture (S4C), déclinés en cinq compétences générales (CG). Pour répondre au souci du vivre ensemble nous mettons l'accent dans ce travail sur le domaine $3 \mathrm{du}$ S4C : la formation de la personne et du citoyen. Cette dernière relève de tous les enseignements. Il s'agit pour l'enseignant d'EPS d'amener les élèves à faire preuve d'esprit critique, à partager des règles, à assumer des rôles et des responsabilités au sein d'un collectif pour réaliser un projet ou remplir un contrat dans le respect de toutes les différences. Cependant, Ott (2015) montre qu'il ne s'agit pas uniquement de mettre en place des situations de coopération, d'échanges, d'interactions pour que les élèves développent des compétences liées au vivre-ensemble. C'est en ce sens que l'enseignant d'EPS mobilisera ses savoirs professionnels afin de favoriser le vivre-ensemble.

Ainsi, questionner le vivre ensemble en contexte interculturel et l'intégrer pour favoriser la construction de compétences en EPS, questionne directement les pratiques pédagogiques des enseignants, les modes de relation et les stratégies d'apprentissage des élèves. Cet enjeu s'inscrit dans une optique plus large d'éducation interculturelle. A ce titre, Wallian (2018) rappelle qu'en éducation et en formation, la dialectique entre enseignement et apprentissage implique une rencontre entre des acteurs aux univers symboliques et aux mondes culturels hétérogènes. Les transformations, fruits de ces interactions, reposent sur une (re)médiation active des savoirs, mais elles questionnent également le poids des contingences et des contextes, qu'ils soient locaux et/ou périphériques, sociaux et/ou historiques, culturels et/ou structurels, discursifs et/ou incarnés. Ainsi il s'avère nécessaire de développer chez tous les enseignants des compétences interculturelles. Selon Potvin, Magnan, \& Larochelle-Audet (2016), ces dernières correspondent à des attitudes d'ouverture à la diversité; des compétences pour inclure le pluralisme dans le projet éducatif; des habiletés à transmettre des valeurs, à résoudre les conflits, à communiquer, quand le cas se présente, avec les parents allophones qui n'ont pas les mêmes valeurs éducatives, les mêmes coutumes, etc.

Si les compétences interculturelles permettent dès lors, de comprendre les normes culturelles et les attentes d'autrui, de les faire interagir efficacement, y compris en dehors de leur environnement d'origine (Hunter, White \& Godbey, 2006), comment les enseignants en EPS les construisent ? Quelles compétences interculturelles mobilisent-ils pour une éducation au vivre ensemble signifiante en contexte interculturel ? En quoi leurs rapports au vivre ensemble peuvent être des freins ou des leviers pour une éducation au vivre ensemble ? Nous cherchons donc dans ce travail à identifier, à travers une étude de cas, les représentations des enseignant-e-s d'EPS relatives au vivre ensemble et les compétences interculturelles qu'ils mobilisent pour le favoriser en EPS en contexte interculturel. En effet les représentations des enseignants sont des constructions à la fois cognitives et sociales. Elles peuvent remplir des fonctions plurielles et, plus particulièrement, selon l'analyse d'Abric (1994), des fonctions de savoir, d'identité, d'orientation et de justification. Nous pensons que le sens que les enseignants accordent au vivre ensemble influe fortement sur leurs actes d'enseignement, qu'il s'agisse de planification d'activités, d'interactions avec les élèves ou d'évaluation.

\section{Méthodologie}

Nous faisons le choix de nous appuyer sur une étude de cas que Merriam (1998) définit comme un moyen d'identifier et d'interpréter plutôt qu'une recherche de validation des hypothèses émises. Cette méthode qualitative permet d'étudier les singularités des individus dans un contexte particulier. Le recueil de données a été réalisé par le biais d'entretiens semi-dirigés et d'une observation participante, auprès de deux enseignants, dans deux classes de 4éme d'un collège REP aux caractéristiques socioculturelles très diverses. Ce collège compte plus d'une trentaine de nationalités différentes. Dans le cadre de cet article, nous exploitons les données relatives aux discours des enseignants. Le choix de cet établissement est lié au fait que le vivre ensemble est un enjeu fort dans les projets d'établissement et d'EPS. Les 
Actes de la 11ème Biennale de l'ARIS : Former des citoyens physiquement éduqués. Axe 2 - Vers une éducation physique de qualité, 10.25518/sepaps20.401

deux enseignants ont plus d'une dizaine d'années d'expérience d'enseignement dont deux pour l'enseignant 1 (E2) et quatre pour l'enseignante 2 (E2) dans cet établissement.

Lors de l'entretien semi-dirigé nous cherchons à recueillir des informations sur la conception d'enseignants d'EPS relative à la diversité culturelle, au vivre ensemble, à leurs prises en compte et leurs mises en œuvre, et des compétences professionnelles nécessaires à leur enseignement. Deux entretiens de 60 minutes ont été menés avec chaque enseignant avant la séquence et après leçon 4. Pour analyser les données recueillies, en référence aux travaux de Baribeau (2009), nous utilisons un protocole d'analyse qui évolue par étapes, « coder, catégoriser, décrire puis modéliser ou théoriser ». Le traitement des données d'entretien s'est donc opéré à partir d’un repérage dans le discours, d'indicateurs sur les valeurs, les croyances des enseignants, leur perception du contexte, les outils didactiques et pédagogiques pour favoriser le vivre ensemble dans leur classe.

\section{Résultats et discussion}

L'analyse des discours des deux enseignants mettent en exergue un rapport au contexte singulier, des conceptions du vivre ensemble en EPS ainsi que des compétences interculturelles différentes.

Les enseignants interrogés définissent leur environnement d'enseignement «comme un contexte regroupant des élèves, majoritairement en situation défavorisées, aux diversités sociales et culturelles multiples » (E1 et E2). Pour eux, beaucoup d'élèves « manquent de ressources et sont en décalage avec le cadre attendu par l'école et les enseignants » (E1). A ce propos, Sève, Vors et Mathé (2018) soulignent que les contextes d'enseignement difficiles concentrent des élèves dont les caractéristiques sociales, scolaires et civiques sont éloignées de la représentation de l'élève idéal. Cet écart entraîne chez les intervenants des difficultés de décider et d'agir. Pour autant, le rapport au contexte diffère selon les enseignants. Ainsi, pour l'enseignant (E1), la diversité culturelle est importante dans l'établissement mais «elle n'influence pas ses contenus d'enseignement, toutefois quand l'occasion se présente, certaines formes traditionnelles (comme en lutte ou en danse) peuvent être évoquées ». Pour lui, « la laïcité et la culture commune sont les repères et la finalité de leur action » (E1). Ce point de vue n'est pas partagé par l'enseignante (E2) qui milite pour une réelle prise en compte de la «diversité culturelle dans ses contenus et son enseignement, notamment dans les groupements en fonction des APSA, ou encore sur le rapport aux autres et au corps qui peut varier et influer sur la pratique des élèves ». Pour elle, il s'avère nécessaire « d'adapter les contenus, le langage et des codes de communication pour permettre à tous les élèves de s'engager ». Ces propos mettent en évidence deux conceptions différentes de l'interculturel. Contrairement à E1, E2 considère la diversité culturelle comme « une ressource à exploiter ».

S'agissant de leur représentation du vivre ensemble, lors de l'entretien, E1 nous affirme que son leitmotiv, « est le respect des règles, des consignes et des autres ». En effet, le respect des règles est au cœur de son enseignement et du vivre ensemble. Selon lui, le vivre ensemble, "c'est d'abord respecter un ensemble de règles communes qui permettront aux élèves de s'exprimer en tant qu'individu dans un collectif » (E1). Il tente de mettre en œuvre le vivre ensemble dans la gestion de la classe, notamment de la formation des groupes et la transmission des consignes. E1 précise qu'il faut faire "preuve de fermeté, d'autorité pour créer un climat de classe agréable et sécurisant ». Quant à E2, le vivre ensemble renvoie "à la notion de communauté, de groupe et se décline par : se mettre au service des autres, coopérer, s'entraider et surtout se respecter, accepter l'autre sans porter de jugement, respecter les règles de vie commune ». Pour elle, le vivre-ensemble relève « de la construction d'un groupe qui partage des règles, qui s'entraide et coopère, et qui accepte la diversité des membres qui le compose ». Il s'apprend par "l'échange et l'interaction dans un cadre régit par des règles co-construites » (E2). Nous constatons ici que les représentations des enseignants sont intéressantes à étudier, puisqu'elles influent directement sur les stratégies d'enseignement et ainsi indirectement sur les apprentissages des élèves (Fives \& Grégoire Gill, 2015). L'E1 s'appuie sur des orientations de valeur (Pasco, Le Bot \& Kermarrec, 2012), qui entraînent un enseignement basé sur la transmission de savoirs, sur une posture de contrôle (Bucheton \& Soulé, 2009). Arraché à une croyance de transmission directe, il met tout en œuvre pour instaurer un 
Diversité culturelle et éducation au vivre ensemble en EPS : quelles compétences interc...

climat de classe calme et propice aux apprentissages. Les croyances de E2, centrées sur l'élève et son action, sont de types constructivistes (Nie \& Lau, 2010). Elles favoriseraient l'engagement, la motivation et l'intérêt des élèves. Ces croyances déterminent et structurent les compétences professionnelles des enseignants. En effet, elles incarnent la manière dont les enseignants considèrent les processus d'apprentissage et d'enseignement (Chan \& Elliott, 2004).

Les résultats montrent également que les deux enseignants mobilisent des compétences professionnelles différentes pour favoriser une éducation au vivre ensemble. L’E1 précise qu'il faut certes «aimer le contexte et les élèves; il ne faut pas d'a priori mais il ne tient pas compte des origines culturelles des élèves ». Ce qui est important pour lui c'est « l'équité, la rigueur, la fermeté et surtout le travail en équipe entre les enseignants pour s'entendre et créer une continuité ». L'E2 souligne que "pour ces élèves souvent en difficultés, la rigueur, l'empathie et la disponibilité sont essentielles aux bonnes relations et au travail de ces élèves ». Pour elle, il faut aussi «faire preuve de patience, d'adaptation, et de répartie. En effet ces élèves sont souvent difficiles à canaliser. Ils peuvent s'embrouiller vite, mais ils sont plein de créativité et de volonté si on s'intéresse à eux, à ce qu'ils sont ». Cette diversité des compétences mobilisées en contexte interculturel peut s'expliquer par le fait que les postures adoptées par les enseignants sont souvent dictées par les valeurs qu'ils souhaitent transmettre et les convictions profondes qu'ils ont vis-à-vis des élèves, de l'enseignement et de l'environnement. La position de ces deux enseignants corroborent les travaux de GrimaultLeprince (2011) qui mettent en évidence l'existence « d'enseignants formalistes » qui défendent une autorité statutaire avec la sanction (E1) et des « enseignants pragmatiques » qui exercent une autorité experte en combinant domination charismatique et négociation (E2). Pour maintenir une atmosphère de confiance, l'E2 semble d'avantage faire preuve de compétences interculturelles, en adoptante une posture plus souple, ouverte à l'autre, tolérante et affective, pour répondre aux besoins des différents élèves.

\section{Conclusion}

Les résultats montrent qu'enseigner en contexte interculturel nécessite souvent une conscience et une distanciation vis-à-vis de sa propre « ethnicité » culturelle, une ouverture à l'autre, une aptitude à adapter ses conceptions et ses comportements dans un groupe. Les différences culturelles, religieuses, ou linguistiques des enseignants et des élèves peuvent être envisagées comme un moyen de se construire librement, afin d'exercer sa citoyenneté. Les résultats révèlent également que les enjeux du vivre ensemble ne sont pas suffisamment identifiés par tous les enseignants. Cela s'explique par l'absence de cadre et de contenus formalisés par l'équipe EPS dans cet établissement et par le fait que, comme tout autre contenus, celui-ci est à l'épreuve de la subjectivité de l'acteur et de son interprétation. Ainsi pour favoriser une éducation au vivre ensemble, enseignants comme élèves, peuvent s'appuyer sur des compétences interculturelles telles que le respect, l'ouverture à l'autre, la reconnaissance des différences, l'empathie, l'écoute, le dialogue, l'entraide et la tolérance. Ainsi nous considérons que, le vivre-ensemble en milieu scolaire, représente davantage que la visée d'une coexistence entre individus et groupes. C'est un projet éducatif, social et politique qui fait de l'esprit critique, de l'exigence de reconnaissance de l'Autre, de l'inclusion du pluralisme dans les contenus et de la recherche du bien-être commun, le socle de la formation de l'élève en tant que personne et futur citoyen.

\section{Bibliographie}

Abric, J-C. (1994). Pratiques sociales et représentations. Paris : PUF.

Baribeau C. (2009). Analyse des données des entretiens de groupe. Recherches Qualitatives, 28(1), 133-148.

Bucheton, D., \& Soulé, Y. (2009). Les gestes professionnels et le jeu des postures de l'enseignant dans la classe : un multi-agenda de préoccupations enchâssées. Education et didactique, 3(3), 29-48.

Chan, K.-W., \& Elliott, R. G. (2004). Relational analysis of personal epistemology and conceptions about teaching and 
Actes de la 11ème Biennale de l'ARIS : Former des citoyens physiquement éduqués. Axe 2 - Vers une éducation physique de qualité, 10.25518/sepaps20.401

learning. Teaching and Teacher Education, 20(8), 817-831.

Dubet, F. (2014). La Préférence pour l'inégalité. Paris : Seuil/La République des idées.

Duhamel, A. (2010). Le « vivre-ensemble » : la citoyenneté et le politique entre conflit et confiance. In F. Jutras (Ed.), L'éducation à la citoyenneté : enjeux socioéducatifs et pédagogiques (pp. 111-130). Québec, QC : Presses de l'Université du Québec.

Duhamel, A., \& Estivalèzes, M. (2013). Vivre-ensemble et dialogue : du programme québécois d'éthique et culture religieuse à la délibération démocratique. McGill Journal of Education, 48(1), 79-98.

Fives, H., \& Gregoire Gill, M. (dir.). (2015). International handbook of research on teachers' beliefs. New York: Routledge.

Grimault-Leprince, A. (2011). La gestion de la classe par les enseignants de collège. Formalise versus pragmatisme. Carrefours de l'éducation, 31(1), 217-235.

Hunter, B., White, G.P., \& Godbey, G. (2006). What does it mean to be globally competent? Journal of Studies in International Education, 10(3), 267-285.

Lamamy-Echard, I. (2016). Vivre Ensemble le Socle, e-novEPS, 11, 3-13.

Lorcerie, F. (2016). Nouvelles proximités dans les collèges publics, Revue Projet, 352, 61-68.

Pasco, D., Le Bot, G., \& Kermarrec, G. (2012). Les orientations de valeur des enseignants d'éducation physique et leur compatibilité avec les objectifs de développement personnel des programmes, Carrefours de l'éducation, 33, 163-181.

Merriam, S. (1998). Qualitative research and case study applications in education. Revised and expanded from "Case study research in education". San Francisco, CA: Jossey-Bass.

Ministère de l'Éducation Nationale, de l'Enseignement supérieur et de la Recherche (2015). Programmes d'enseignement du cycle des apprentissages fondamentaux (cycle 2), du cycle de consolidation (cycle 3) et du cycle des approfondissements (cycle 4). Bulletin Officiel spécial, 11, 26 novembre 2015. NOR : MENE1526483A, arrêté du 9-11-2015 - J.O. du 24-11-2015 MENESR - DGESCO MAF.

Nie, Y., \& Lau, S. (2010). Differential relations of constructivist and didactic instruction to students' cognition, motivation, and achievement. Learning and Instruction, 20(5), 411-423.

Ott, L. (2015). Peut-on transmettre les valeurs essentielles du vivre ensemble sans les donner à vivre ?. Empan, $10(4), 24-31$.

Potvin, M., Magnan, M-O., \& Larochelle-Audet, J. (2016). La diversité ethnoculturelle, religieuse et linguistique en éducation. Théorie et Pratique. Montréal : Fidès Éducation.

Sève, C., Vors, O., \& Mathé, P. (2018). Les contextes difficiles. Paris : Éditions Revue EPS.

Wallian, N. (2018). Interventions éducatives et médiation(s). Contextes insulaires, cultures diverses, explorations plurielles. Berne : Peter Lang.

PDF automatiquement généré le 2021-10-07 19:11:46

Url de l'article : https://popups.uliege.be/sepaps20/index.php?id=401

Publié par ULiège Library en Open Access et distribué suivant les termes et les conditions de la licence CC-BY 
Diversité culturelle et éducation au vivre ensemble en EPS : quelles compétences interc...

(https://creativecommons.org/licenses/by/4.0/deed.fr) 